\title{
Characterizing multiple exogenous and endogenous small RNA populations in parallel with subfemtomolar sensitivity using a streptavidin gel-shift assay
}

\author{
H. ALEXANDER EBHARDT ${ }^{\mathbf{1}}$ and PETER J. UNRAU \\ Department of Molecular Biology and Biochemistry, Simon Fraser University, Burnaby, British Columbia, V5A 1S6 Canada
}

\begin{abstract}
Here we present a simple and inexpensive gel-shift assay for the detection and quantification of small RNAs. The assay is at least 5-10 times more sensitive than a conventional Northern, and is highly scalable. Total RNA is first size purified to enrich the desired size range, phosphatase treated, and then radiolabeled to high specific activity using polynucleotide kinase. The resulting RNA stock is then hybridized to an excess of biotinylated DNA probe oligonucleotide, prior to mixing with streptavidin and loading on a native gel. The amount of supershifted material was proportional to the amount of labeled target RNA in the sample. We applied this method to verify sequencing data originally obtained from a four-point comparison study on the effect of endogenous expression of HC-Pro on Y-satellite/cucumber mosaic virus infection in tobacco plants. The results of the streptavidin gel-shift assay were consistent with the concentrations of small RNA infected plants inferred by our original cloning data, and rapidly provided information about the relative concentration of a number of viral and endogenous small RNAs. Further straightforward improvements to this simple methodology might be expected to improve the methods sensitivity by as much as another 10-fold.
\end{abstract}

Keywords: streptavidin gel shift; viral small RNA; endogenous small RNA; quantification

\section{INTRODUCTION}

High-throughput sequencing has revolutionized the study of small RNA expression levels and provides a "digital" readout of the relative RNA concentrations found in an RNA sample by counting the number of each type of small RNA sequenced in each sample (Rajagopalan et al. 2006; Mi et al. 2008; Morin et al. 2008). Despite the power of this new approach, there are many biochemical reasons why relative RNA concentrations inferred by sequencing might disagree with reality. As the workup of RNA for sequencing involves the ligation of adaptor sequences to the $5^{\prime}$ and $3^{\prime}$ termini of the library members, any sequence modification that interferes with the ligation efficiency might be expected to bias sequencing results. In fact, the ubiquitous terminal $3^{\prime}$ methylation of plant small RNAs significantly inhibits

${ }^{1}$ Present address: 3-16 Medical Sciences Building, Department of Biochemistry, University of Alberta, Edmonton, Alberta, T6G 2H7 Canada.

Reprint requests: Peter J. Unrau, Department of Molecular Biology and Biochemistry, Simon Fraser University, 8888 University Drive, Burnaby, BC, V5A 1S6 Canada; e-mail: punrau@sfu.ca; fax: (778) 782-5583.

Article published online ahead of print. Article and publication date are at http://www.rnajournal.org/cgi/doi/10.1261/rna.1235109. ligation in plants. The endogenous expression of HC-Pro in tobacco led to an unexpected enrichment in viral small RNAs upon sequencing that resulted from a decrease in the methylation of exogenous small RNA (Ebhardt et al. 2005; Li et al. 2005; Yang et al. 2006; Horwich et al. 2007). There is therefore a need for an unbiased methodology to quickly, cheaply, and sensitively measure the relative concentrations of small RNAs found in a biological sample.

Traditionally, RNA levels have been measured by using a Northern blot (Alwine et al. 1977, 1979). Since the probe is labeled, the Northern is best suited for comparing RNA expression levels between distinct samples that are loaded onto the same blot. This limits the ability of the method to measure the relative concentration of distinct RNAs within the same sample and requires the blot to be stripped and reprobed. Microarrays are ideal for the comparison of relative RNA concentrations within a sample, but microarrays are costly and tend, due to hybridization kinetics, to suffer from a relatively low dynamic range (Zhang et al. 2005; Eklund et al. 2006; MAQC Consortium et al. 2006). Methods based on quantitative PCR are likely to be very sensitive but require multiple enzymatic steps and multiple pairs of oligonucleotides to achieve results. This may 
ultimately bias the quantification of small RNA concentrations. The miR-Q protocol, for example (SharbatiTehrani et al. 2008), is based on annealing a heterohexamer oligonucleotide to the $3^{\prime}$ terminus of small RNAs, followed by reverse transcription with a second primer specific to the sequence of interest. While each of the methods just discussed have particular strengths, they are not simultaneously cheap, sensitive, and fast.

To counter these limitations we developed a novel streptavidin gel-shift assay that makes possible the direct comparison of multiple small RNA expression levels within a particular sample using a single gel. This simple approach uniformly labels a small RNA library and then ensures efficient hybridization by using an excess of a probe sequence. The method requires no specialized equipment or enzymatic reactions, and has a demonstrated ideal detection limit in the low attamole range in our hands. While sensitivity is lower with real samples, we have routinely obtained sensitivities that are $5-10$ times better than for a conventional Northern and further simple improvements to the methodology might be expected to realize a further factor of 10 in sensitivity. We apply the method to quickly verify relative small RNA levels that were inferred from sequencing Y-satellite (Y-Sat) and cucumber mosaic virus $(\mathrm{CMV})$ infected tobacco plant libraries.

\section{RESULTS AND DISCUSSION}

\section{Streptavidin gel-shift assay}

We devised a method in which size fractionated small RNA is first $5^{\prime}$ phosphorylated using $\left[{ }^{32} \mathrm{P}\right]-\gamma \mathrm{ATP}$ and then hybridized to biotinylated DNA oligonucleotides complementary to particular target small RNA. As a result of the biotin label, the nucleic acid duplex forms an extremely stable complex with streptavidin that migrates in a native gel significantly slower than unhybridized RNA. This shift allows the accurate determination of a particular RNA concentration relative to the total small RNA concentration. Figure 1A illustrates the annealing of a biotinylated DNA probe to a radiolabeled RNA (Fig. 1A, lane 1). Adding streptavidin to the hybridized dimer shifts the tagged complex substantially upward on the gel, as seen in Figure $1 \mathrm{~A}$, lane 2 . To test the sensitivity of this method, we size fractionated tobacco plant small RNA using a preparative polyacrylamide gel. The recovered RNA then was treated with calf alkaline phosphatase (CIP) to remove $5^{\prime}$ phosphates which could otherwise interfere with labeling efficiency and then radiolabeled with polynucleotide kinase (PNK). This RNA was held at a fixed concentration and spiked with a synthetic radiolabeled RNA called 24.11 in a dilution series from $9.6 \mathrm{fmol}$ to 77 amol. The radiolabeled RNA was hybridized to a biotinylated DNA probe specific for the 24.11 RNA. The resulting mixture was then heated and cooled prior to mixing with an excess of streptavidin. On a $10 \%$ native gel, the shifted complexes were separated as seen in Figure 1A, lanes 3-6. The detection limit of the synthetic RNA 24.11 was between $1.9 \mathrm{fmol}$ and 384 amol. This threshold was set by the small but appreciable amount of radioactivity present throughout the lanes of the native gel. This smearing presumably resulted from the small RNA radiolabeling protocol, which uses an excess of $\left[{ }^{32} \mathrm{P}\right]-\gamma \mathrm{ATP}$.

To establish the detection limit under ideal conditions, we gel purified the 24.11 RNA prior to mixing it with unlabeled plant small RNA. The dilution series were again analyzed by $10 \%$ native PAGE (Fig. 1B, lanes 1-6). The detection limit in this experiment was considerably improved and was found to be in the $\sim 10$ amol range. Interestingly, removing the plant RNA from the experiment did not appreciably alter this sensitivity (Fig. 1B, lanes 712). This 40 - to 100 -fold increase in sensitivity indicates that further improvement in small RNA labeling, such as removal of unincorporated $\left[{ }^{32} \mathrm{P}\right]-\gamma \mathrm{ATP}$ by desalting with a spin column prior to the gel-shift assay, are likely to further improve the sensitivity of our straightforward methodology.

Even without such a step, the gel-shift protocol was significantly more sensitive than a Northern blot. Dilution series identical to those used in Figure 1, A and B, were transferred to a positively charged membrane, blocked, and then probed with the same DNA oligonucleotide used in the gel-shift experiments. Figure 1C, lanes 1-6, show in the top panel the radiolabeled 24.11 probe being detected in the low fmole range. The same membrane was stripped and reprobed using a MIR166 DNA probe. The signal for MIR166 stayed constant as expected; reflecting the fixed concentration of plant small RNA in each lane. When total plant small RNA was omitted from the Northern, the 24.11 probe showed increased sensitivity in contrast to the gelshift methodology, which appears less biased in this respect. The MIR166 probe in Figure 1C, lanes 8-13, was blank in these lanes, as expected due to the absence of plant RNA in these lanes.

Comparing Figure 1, A and C, indicates that the gel-shift methodology is $5-10$ times more sensitive than a conventional Northern analysis. As the method is intrinsically able to detect RNA concentrations in the low attamole range (Fig. 1B), we believe that further improvements to the methodology should ultimately be able to improve sensitivity further.

\section{Case study: Small RNAs from CMV and its Y-satellite-infected plants}

A four-point comparison study was used to characterize $\mathrm{CMV} / \mathrm{Y}$-Sat infection of tobacco plants as a function of endogensous HC-Pro expression. The samples in the study were: s1: wild type, s2: $\mathrm{HC}-\mathrm{Pro}^{+}$, s3: CMV/Y-Sat ${ }^{+}$, and s4: $\mathrm{HC} \mathrm{Pro}^{+}, \mathrm{CMV} / \mathrm{Y}-\mathrm{Sat}^{+}$(Ebhardt et al. 2005). Small RNAs isolated from individual plants were size fractionated and 
A
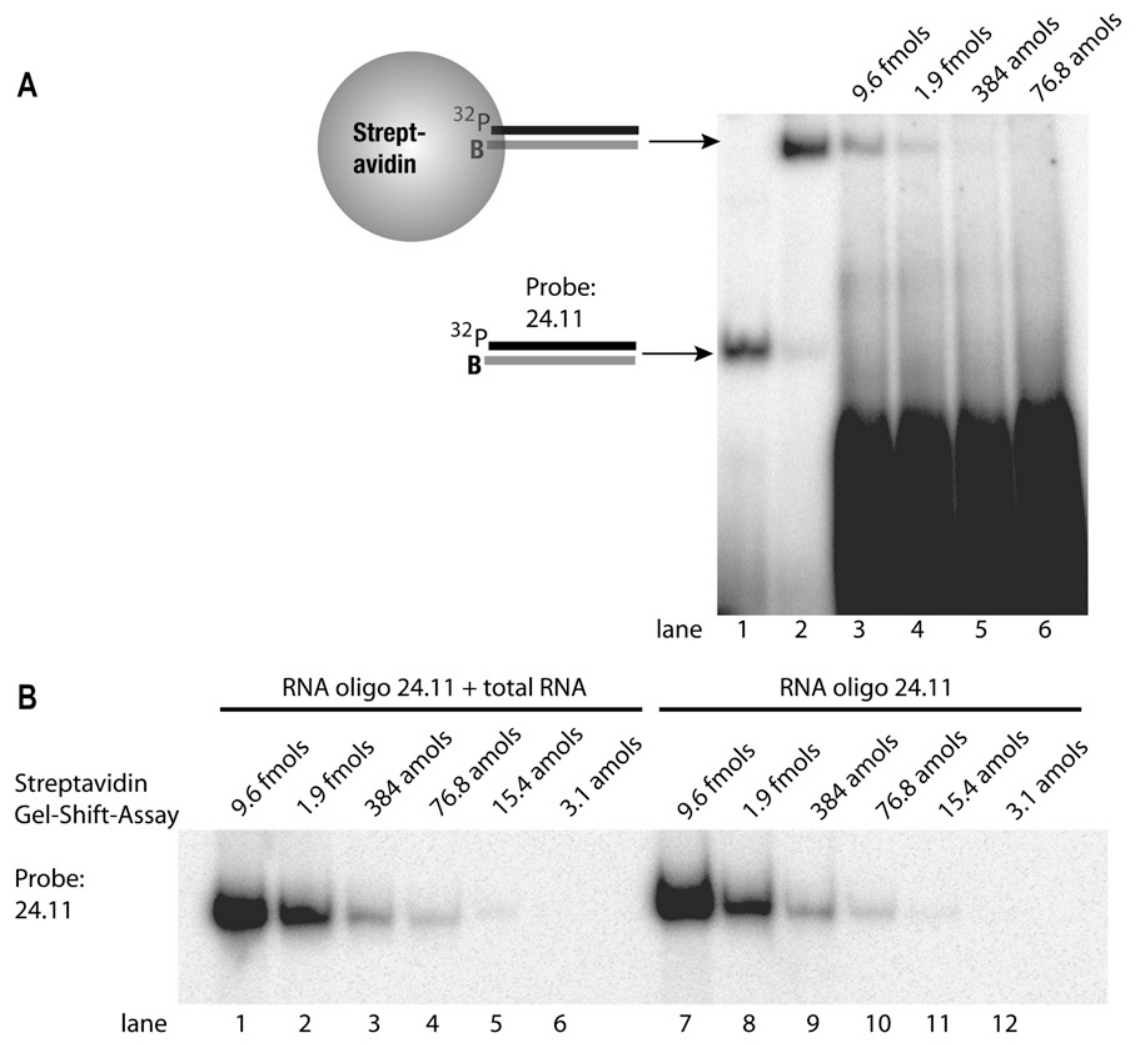

C
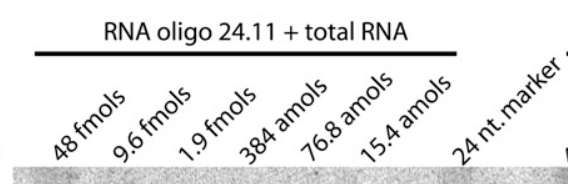

RNA oligo 24.11

Northern Hybridization

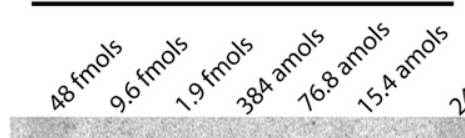

Probe:

24.11
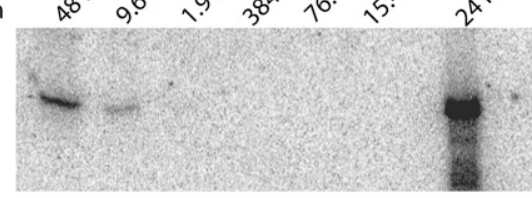

Probe:

MIR166

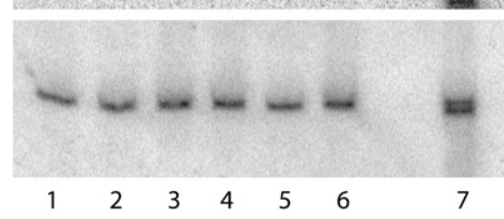

lane

2

FIGURE 1. Comparing the sensitivity of a Streptavidin gel-shift assay to a Northern hybridization. $(A)$ In an autoradiogram of a $10 \%$ native PAGE, a complementary $3^{\prime}$ biotinlabeled probe was annealed to a $5^{\prime}$ radiolabeled RNA (lane 1). The annealed duplex migrates significantly slower when it is bound to streptavidin (lane 2). Lanes 3-6 are serial dilutions of a synthesized RNA that was added to a pool of size fractionated small RNAs prior to appending the $5^{\prime}$ radiolabel. Following the phosphorylation reaction and kinase inactivation, the $3^{\prime}$ biotin-labeled probe was annealed and streptavidin added to induce a gel shift. The assay using gel-purified RNA detects as little as 384 amol of radiolabeled RNA (lane 4). (B) Autoradiogram of a $10 \%$ native PAGE where 5' radiolabeled RNA is titrated in fivefold dilutions with (lanes 16) or without (lanes 7-12) nonradiolabeled total plant RNA (size purified previously on a denaturing gel). The sensitivity of the streptavidin gel-shift assay under these ideal conditions is limited to the detection limit of the phosphorimaging system, which is between 15 and 3.1 amol. (C) Autoradiogram showing a Northern hybridization experiment under identical conditions to $B$. In $C$ on top, a probe for the artificial RNA is added and in the bottom panel a probe for MIR166 was added. The sensitivity of the Northern is at least 125-fold lower than the streptavidin gel-shift assay in ideal conditions. adaptors ligated onto their $3^{\prime}$ and $5^{\prime}$ termini. Subsequently, the constructs were reverse transcribed, cloned, and sequenced. The resulting sequences were analyzed by a software package called Ebbie (Ebhardt et al. 2006). Ebbie searches the sequencing file for the two adaptor sequences and excises the sequence corresponding to the small RNA. Then, the new small RNA sequence is compared with all previously cloned small RNAs by a locally installed BlastN search. The advantage of the automated approach is the elimination of human error and the instant comparison of new RNA sequences to sequences previously deposited into the project database. Using this approach a total of 698 small RNAs were classified, with 213 being of viral origin (see Supplemental Data). In all, 165 of viralderived small RNAs were from the Y-Sat genome and 48 from the tripartite $\mathrm{CMV}$ genome. As the tripartite CMV genome consists of 8623 nucleotides (nt) and Y-Sat genome of $369 \mathrm{nt}$, we had an $\sim 90$-fold higher coverage of the Y-Sat genome by small RNAs than the CMV genome. This can only partially be accounted for by the seven- to 10 -fold higher concentration of Y-Sat RNA in infected plants (Takanami 1981). The remaining sequences, in the absence of a complete tobacco genome, presumably correspond to RNAs derived from the host system. The more highly expressed of these small RNAs were clustered into 32 distinct groups that were classified into five clades: the NULL-clade, microRNA, silencing RNA clade, rRNA clade, and tRNA clade (Supplemental Table 1).

\section{Endogenous small RNA}

We used the streptavidin gel-shift assay to study some of the endogenous small RNAs discovered in our four-point study. Fresh small RNA from adult leaves of tobacco plants (Wisconsin38) was extracted and radiolabeled. The resulting RNA was then used to test the expression of 10 RNAs simultaneously in a single gel. Figure 2 shows the formation of a shifted RNA-DNA-streptavidin complex, with a radiolabeled synthetic 
RNA (Fig. 2, lane 1), that is then hybridized to a biotinylated DNA to form a duplex (Fig. 2, lane 2), which is then shifted up the native gel by the addition of streptavidin (Fig. 2, lane 3). Lanes 4 to 8 show gel shifts induced with various endogenous microRNA probes. Notably, MIR166 does not show a strong signal in mature leaves when compared with RNA isolated from young leaves in our four-point comparison study. This observation is consistent with Jung and colleagues (Jung and Park 2007), who established that the MIR165/MIR166 family is involved in lateral meristem formation, leaf polarity, and vascular development. Figure 2, lanes 10-14, shows the streptavidin shift when using complementary probes for various small RNA groups identified by Ebbie, as discussed above. A negative control in Figure 2, lane 9, consisting of a random sequence probe remained blank as expected.

\section{Exogenous small RNA}

Besides endogenous small RNAs, exogenous small RNAs stemming from the infection of tobacco plants with Y-Satellite and its helper virus CMV were found in the small RNA cloning data. Small RNAs cloned from Y-Sat were aligned

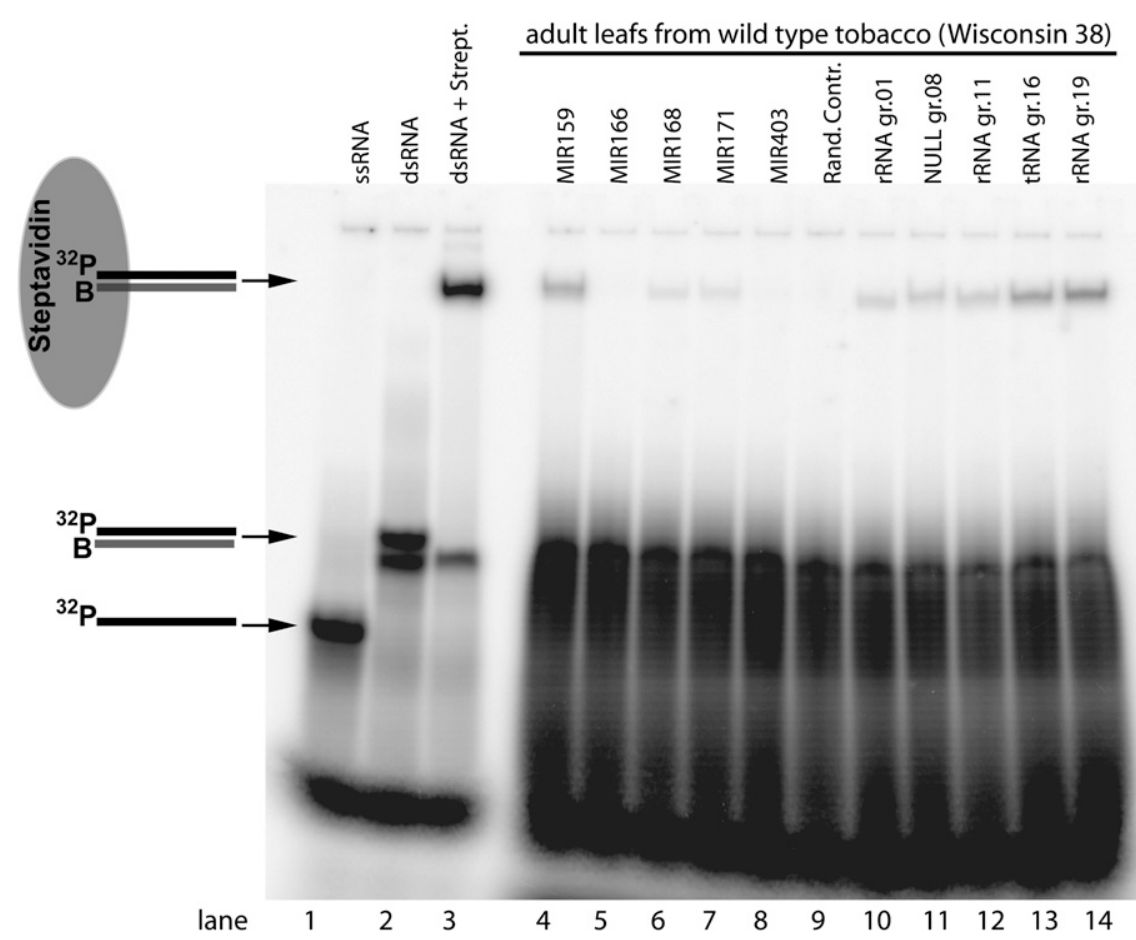

FIGURE 2. Probing for micro-RNAs and ribosomal and tRNA groups commonly found in sequencing results. Autoradiogram of a 10\% native gel: lanes 1-3 demonstrating the stages of the streptavidin gel-shift assay: single-stranded radiolabeled RNA, RNA annealed to biotinlabeled probe and streptavidin annealed to the DNA-RNA duplex. RNA was extracted from fully grown leafs of wild-type tobacco plants (strain: Wisconsin38), size fractionated by denaturing PAGE and 5' radiolabeled using polynucleotide kinase. Various 3' biotin labeled DNA probes (microRNAs, a negative control, and various rRNA groups) were annealed to the radiolabeled RNA and streptavidin added prior to being loaded on the gel. to the Y-Sat sequence and in $\mathrm{HC}^{-P r o}{ }^{+}$plants $\mathrm{Y}$-Sat small RNAs were found that spanned the entire Y-Sat genome (Fig. 3A, green lines). While 65\% were derived from the $(+)$ strand, a considerable number of sequences were derived from the (-) strand (35\% or 46/131). Only rarely were solitary small RNAs found and typically small RNAs were found in strongly overlapping clusters. These clusters were found in a staggered pattern with high expression on the $(+)$ strand alternating with strong expression on the $(-)$ strand in the middle of the Y-Sat genome. Interestingly, at the $5^{\prime}$ terminus this staggered pattern disappears and the small RNAs from both strands occur in phase with approximately equal amounts of $(+)$ and $(-)$ small RNA being produced in the same register (Fig. 3A, $\sim 20-40 \mathrm{nt}$ ). At the other end of the genome, significant levels of small RNA are only seen after moving $\sim 35 \mathrm{nt}$ in from the $3^{\prime}$ terminus of the Y-Sat genome.

All small RNAs from the CMV genome had a length between 20 and 24 nts and are listed in the Supplemental Data; the majority, 36 out of 48 CMV small RNAs, originated from CMV RNA III (21 out of 48 sequences were derived from HC-Pro ${ }^{+} \mathrm{CMV} / \mathrm{Y}-$ Sat infected samples). Of those small RNAs mapping to the CMV III genome, 24 mapped to the $(+)$ strand and 12 mapped to the (-) strand (see Supplemental Fig. 1). The remaining $12 \mathrm{CMV}$ small RNAs mapped to CMV RNA I (3 to $[+], 1$ to [-] strand) and CMV RNA II (6 to $[+], 2$ to $[-]$ strand). The relative amount of small RNA from the $(+)$ and $(-)$ strand of CMV RNA III is consistent with the higher levels of (+) strand expression that is known to exist (Sivakumaran and Kao 1999) from the rolling circle replicative process (Dressler 1970; Modahl and Lai 1998) that is thought to produce these genomic elements.

We have previously demonstrated that changes in terminal methylation (such as are induced by HC-Pro expression) affect RNA ligation efficiency (Ebhardt et al. 2005). To ensure that our cloning data reflected the distribution of Y-Sat small RNAs found in the total small RNA population, six $3^{\prime}$ biotin-labeled DNA sequences were designed to probe both the sense and antisense of three $\mathrm{Y}$-Sat genomic regions (Fig. 3A, positions 76-99, 173193, 225-247). For the yellowing region of Y-Sat, Figure 3A, positions 173-193, a ratio of two antisense to 12 sense was observed from the cloned data. The intensities in the gel shift also have a 
A
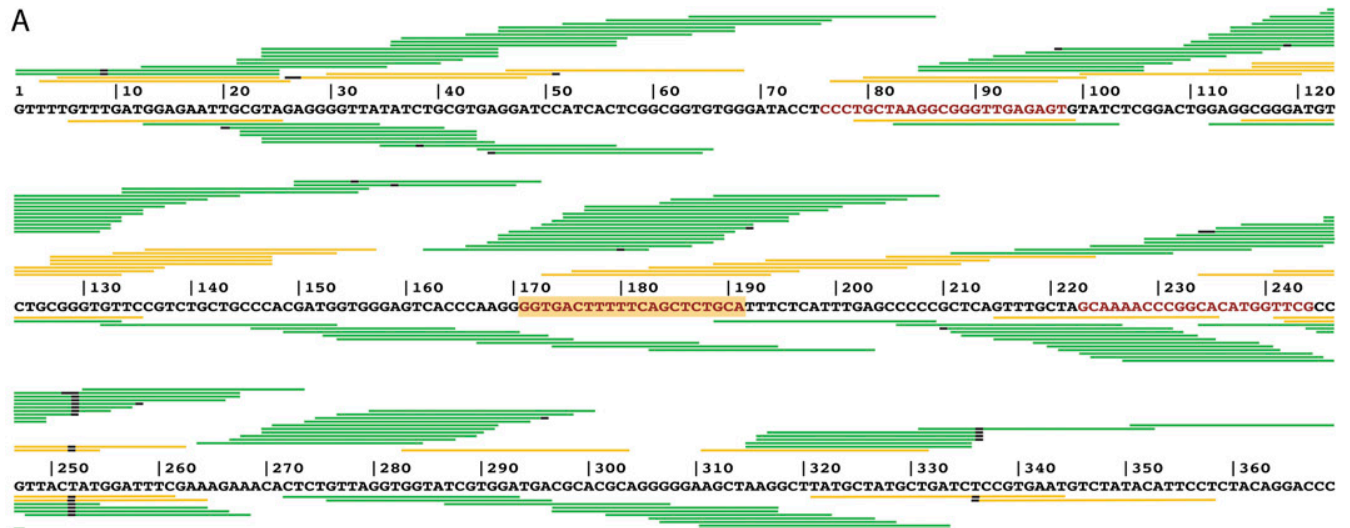

Legend: \#5 HC-Pro(+) CMV/Y-Sat(+) \#6 HC-Pro(-) CMV/Y-Sat(+)

B

tobacco plants expressing HC-Pro

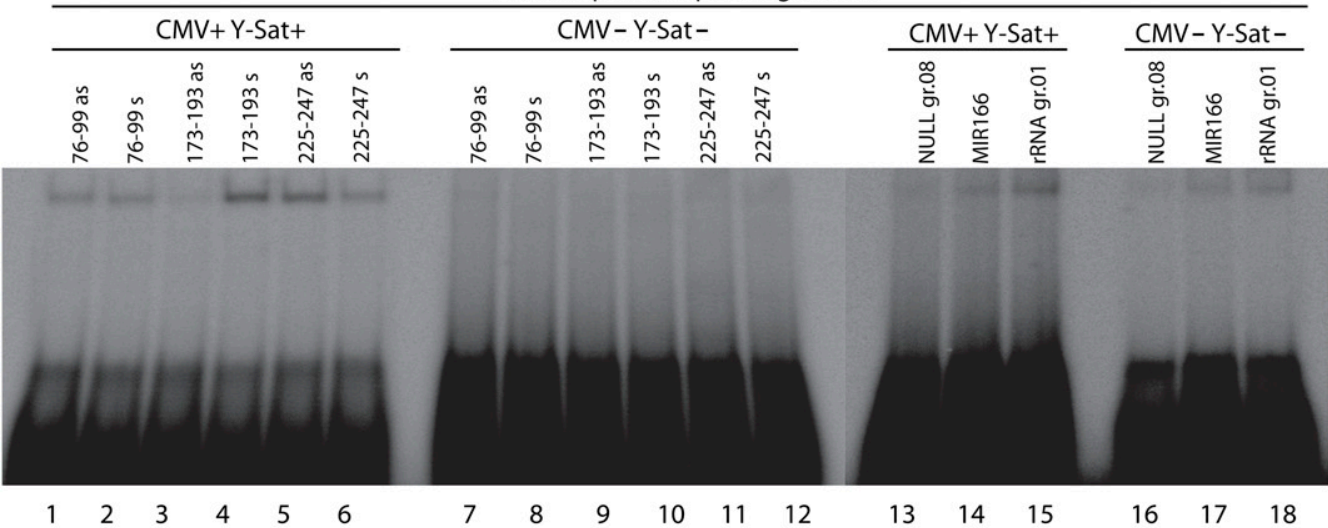

C

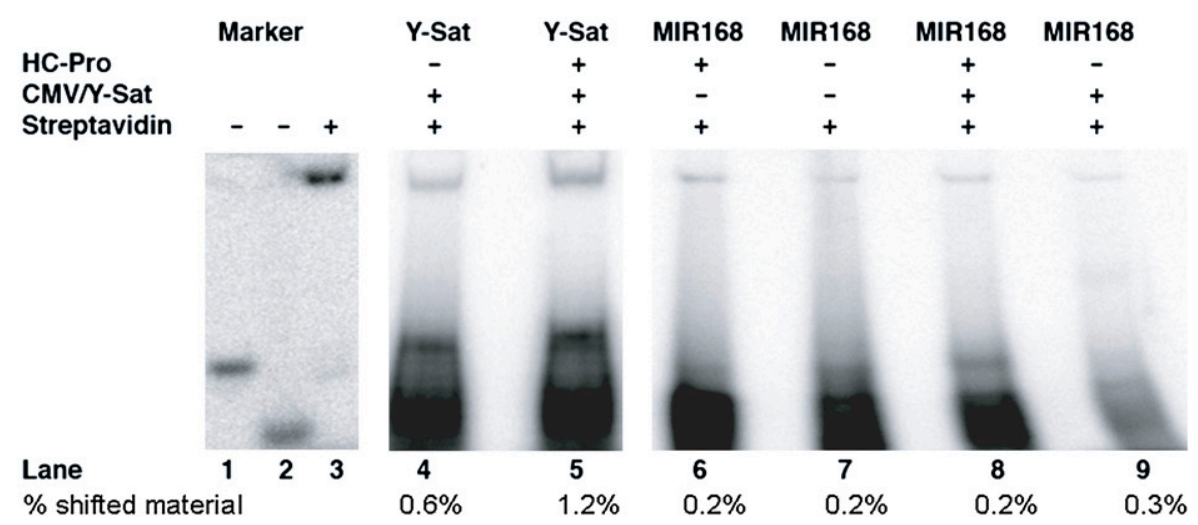

FIGURE 3. Small RNAs from CMV's Y-Satellite. (A) In black letters is the Y-Sat genome and its numbering. Red letters within the Y-Sat genome refer to the yellowing region (positions 171-191) and the necrosis region (positions 223-244) (Masuta et al. 1993; Sleat et al. 1994). Green bars represent small RNA cloned from $\mathrm{HC}-\mathrm{Pro}^{+}$plants, whereas yellow bars are small RNA cloned from $\mathrm{HC}^{-P r o}{ }^{-}$plants. Colored bars above the Y-Sat genome are from the sense (+) strand; colored bars below the Y-Sat genome align to the antisense (-) strand. If there is a small black line within a colored line it represents a mismatch to the aligned viral genome. $(B)$ Autoradiogram of a streptavidin gel-shift assay probing size purified plant small RNA with six 3' biotin tagged DNA oligonucleotides whose sequences are the reverse complement of the indicated regions (s: plus strand, as: antisense) of the Y-Sat genome. Small RNAs from HC-Pro ${ }^{+} \mathrm{CMV} / \mathrm{Y}_{-} \mathrm{Sat}^{+}$plants (lanes 1-6) and HC-Pro ${ }^{-} \mathrm{CMV} / \mathrm{Y}-\mathrm{Sat}{ }^{+}$plants (lanes 7-12) were probed. As controls, both plant samples were also probed for NULL group 08, MIR166 and rRNA group 01 (lanes 13-18). (C) Comparing small RNA ratios of exogenous and endogenous small RNAs. Autoradiogram of a 10\% native gel: lanes 1-3 show a 5' radiolabeled RNA oligo hybridized to a complementary DNA (lane 1), single-stranded 5' radiolabeled RNA oligo (lane 2), and 5' radiolabeled RNA hybridized to its complementary 3' biotin-labeled DNA oligonucleotide together with streptavidin (lane 3). Lanes 4 and 5 probe for Y-Sat (positions 173-193, plus strand) in $\mathrm{HC}-\mathrm{Pro}^{-}$and $\mathrm{HC}-\mathrm{Pro}^{+}$plants. Lanes 6-9 probe for MIR168 in all tobacco plants in the four-point comparison study. The percentages of material shifted to the total material are given below each lane. 
similar ratio of 1 to 9 , respectively (Fig. 3B, lanes 3,4). The Y-Sat region from 225 to 247 has a ratio of six antisense to four sense, as seen in the cloning data as well as in the native gel-shift assay; the gel-shift ratio was $\sim 2$ to 1 (Fig. 3B,

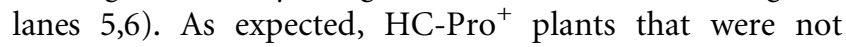
infected with $\mathrm{CMV} / \mathrm{Y}$-Sat failed to produce a gel shift using Y-Sat-specific probes (Fig. 3B, lanes 7-12). Judging from the cloning data, the sense and antisense probe for Y-Sat, Figure 3A, positions $76-99$, should have nearly equal intensities in the autoradiogram of the native gel-shift counting sequences that fully overlapped with this region by $14 \mathrm{nt}$ or more. This was indeed the case (Fig. 3B, lanes 1,2 ) although overall expression as judged by gel shift was higher than anticipated based on our sequencing results. Samples were also probed with three nonviral 3' biotinlabeled oligonucleotides that probed for common endogenous small RNAs (Supplemental Table 1, NULL group 08, MIR166, rRNA group 01) as controls (Fig. 3B, lanes 1318). As the percentage shift observed between infected and uninfected plants for these samples was quite similar we concluded that endogenous small RNA production was not radically different as a result of CMV/Y-Sat infection.

These probes allowed a comparison of viral small RNA levels relative to endogenous small RNA expression levels in a range of plant backgrounds. Figure 3C shows the autoradiogram of a streptavidin gel-shift assay probing for exogenous Y-Sat small RNA (+) strand from HC-Pro ${ }^{+/-}$ plants from the yellowing region (Fig. 3A, Y-Sat positions 173-193) relative to endogenous microRNA (Fig. 3C, MIR168) expression levels. In $\mathrm{HC}^{-P_{0}{ }^{-} \mathrm{CMV} / \mathrm{Y}_{-} \mathrm{Sat}^{+}}$ plants, $0.6 \%$ of the total population of small RNAs hybridized to the Y-Sat 173-193 region probe (Fig. 3C, lane 4). In $\mathrm{HC}-\mathrm{Pro}^{+} \mathrm{CMV} / \mathrm{Y}-\mathrm{Sat}^{+}$, this ratio doubled to $1.2 \%$ (Fig. 3C, lane 5). Considering that this positive strand region samples $\sim 1 / 35$ (the satellite has $\sim 740 \mathrm{nt}$ of plus and minus strand sequences and the probe is 21-nt long) of the available Y-Sat sequence, these numbers agree very well with the $23 \%$ and $57 \%$ observed in the cloning results discussed earlier. The results also show that the endogenous MIR168 was expressed evenly at $0.2 \%$ of the total small RNA population in all four of the tobacco plant types (Fig. 3C, lanes 6-9) and was not strongly perturbed by either viral infection of HC-Pro expression. The observation that Y-Sat-derived small RNAs in HC-Pro ${ }^{+}$plants are expressed at a higher level than in $\mathrm{HC}^{-} \mathrm{Pro}^{-}$is consistent with earlier experiments by Wang et al. (2004) using Northern analysis.

\section{CONCLUSION}

The streptavidin-based gel-shift assay described here takes advantage of the Watson-Crick base pairing between small RNAs and biotin tagged DNA probes. Compared with Northern analysis, our streptavidin gel-shift assay has two major advantages. First, Northern hybridization can only be used with a single radiolabeled probe sequence at a time.
As seen in Figure 2, 10 small RNA expression levels were simultaneously analyzed by gel shift. The same experiment using Northern hybridization would have taken ten separate hybridizations and the relative concentrations of each RNA would have been difficult to determine. Second, the detection limit of Northern is at least 5-10 times worse than for the streptavidin gel-shift assay. We demonstrate that further gains in sensitivity and speed of the method are possible and that detection in the low attomole range is technically possible. This might be achieved by making use of FPLC to size purify small RNA from total RNA preparations and after labeling (Kim et al. 2007). We note that a major assumption of this method is that the small RNA population is, in fact, uniformly labeled by polynucleotide kinase. Fortunately, any RNAs possessing either $5^{\prime}$ phosphates or hydroxyls should be uniformly processed by PNK as long as RNA is initial phosphatase treated prior to labeling.

\section{MATERIALS AND METHODS}

\section{Small RNA isolation procedure}

A sample lacking large RNA fractions is essential with this protocol. The HC-Pro ${ }^{+}$and $\mathrm{HC}-\mathrm{Pro}^{-}$tobacco plants are described by Wang et al. (2004). For the streptavidin gel-shift method development, RNA was isolated from $4 \mathrm{~g}$ of tobacco plant leafs (Wisconsin 38). The leaf tissue was ground into fine powder with mortar and pestle in the presence of liquid nitrogen and sand (Ottawa Standard). The powder was transferred to a 14-mL centrifuge tube (Sarstedt) and suspended quickly in $4 \mathrm{~mL}$ extraction buffer (100 mM LiCl, 1\% SDS, $10 \mathrm{mM}$ EDTA, 100 $\mathrm{mM}$ Tris at $\mathrm{pH} 9$ ), $4 \mathrm{~mL}$ of phenol, and $4 \mathrm{~mL}$ of chloroform. The mixture was constantly inverted for $20 \mathrm{~min}$ at room temperature. After incubation, the tube was spun down in a Sorvall SS-34 rotor and the supernatant transferred to a new $14 \mathrm{~mL}$ centrifuge tube to which $4 \mathrm{~mL}$ of chloroform were added for another extraction. Following centrifugation of the second chloroform extraction, the supernatant was transferred into a new $14 \mathrm{~mL}$ centrifuge tube, $\mathrm{NaCl}$ was added to a final concentration of $300 \mathrm{mM}$, mixed, and $10 \mathrm{~mL}$ of anhydrous ethanol were added to precipitate the nucleic acids overnight at $-20^{\circ} \mathrm{C}$. Smaller amounts of material are most easily prepared using the Trizol reagent (Invitrogen) as described by the manufacturer's protocol. Size fractionation of small RNAs (18-30 nt) was achieved by gel extraction using a 15\% preparative polyacrylamide gel loading 3-6 $\mu$ g of total RNA per square mm of gel-well area in formamide loading dye. To visualize the small RNA during this procedure, a small fraction of isolated RNA was $5^{\prime}$ end radiolabeled using PNK. Small RNAs were eluted from polyacrylamide gel slices overnight in $300 \mathrm{mM} \mathrm{NaCl}$ at $4^{\circ} \mathrm{C}$. The resulting RNA was precipitated by the addition of $12.5 \mu \mathrm{g} / \mathrm{mL}$ glycogen (Ambion) and $2.5 \mathrm{vol}$ of anhydrous ethanol. Samples were placed at $-96^{\circ} \mathrm{C}$ for $60 \mathrm{~min}$ before pelleting the labeled RNA at $12,000 \mathrm{~g}$ for $30 \mathrm{~min}$.

\section{Small RNA labeling}

For high specific activity, small RNA populations were first dephosphorylated using alkaline phosphatase, calf intestinal 
(CIP) from New England Biolabs according to the manufacturer's specifications. To remove CIP, the small RNAs were extracted twice using phenol-chloroform before being precipitated for a second time. The resulting dephosphorylated RNA was $5^{\prime}$ endlabeled at $37^{\circ} \mathrm{C}$ for $15 \mathrm{~min}$ using $0.66 \mu \mathrm{M}\left[\gamma^{-}{ }^{32} \mathrm{P}\right] \mathrm{ATP}(6000 \mathrm{Ci} / \mathrm{mmol}$ specific activity, Perkin-Elmer), $100 \mathrm{mM} \mathrm{NaCl}, 10 \mathrm{mM} \mathrm{MgCl}_{2}, 1 \mathrm{mM}$ DTT, $50 \mathrm{mM}$ Tris. $\mathrm{HCl}$ ( $\mathrm{pH} 7.9$ ), and 0.5 units/ $\mu \mathrm{L}$ PNK (New England Biolabs), PNK was heat killed at $65^{\circ} \mathrm{C}$ for $20 \mathrm{~min}$.

\section{Gel-shift assay}

An aliquot of the radiolabeled plant RNA was then mixed with 3' biotinylated DNA (typically, $2.5 \mu \mathrm{M}$ ) in $50 \mathrm{mM} \mathrm{NaCl}$ and $20 \mathrm{mM}$ Tris-borate at $\mathrm{pH}$. The mixture was heated to $90^{\circ} \mathrm{C}$ for $1 \mathrm{~min}$, slowly cooled to room temperature, and then incubated with an excess of streptavidin (Sigma) for $5 \mathrm{~min}$. The streptavidin-shifted RNA:DNA duplexes were then separated away from other small RNA species in a $10 \%$ native polyacylamide gel containing $50 \mathrm{mM}$ $\mathrm{NaCl}$ and $90 \mathrm{mM}$ Tris-borate at $\mathrm{pH}$ 8. The native PAGE was performed in the cold room and the gel exposed to a Fuji digital PhosphorImager screen at $4^{\circ} \mathrm{C}$. Freezing of native gels during exposure to PhosphorImager screens is not advised, as the native gels will easily crack. The gel's autoradiogram scanned using Typhoon Storm 820 and analyzed using Molecular Dynamics Image Quant.

\section{Biotin probe synthesis}

The following DNA oligonucleotides with a terminal biotin $\left(3^{\prime}-\right.$ BiotinTEG-CPG, Glen Research) and complementary to particular small RNAs were synthesized using standard DNA phosphoramidite chemistry on an ABI 392 machine and were used for the streptavidin gel-shift assay ("B" indicates 3'-BiotinTEG-1000 Angstrom CPG, Glen Research):

MIR166 probe, 5'-GGGGAATGAAGCCTGGTCCGATTB-3'; MIR168 probe, 5'-GTCCCGACCTGCACCAAGCGATTB-3'; Y-Sat probe (nucleotides 193-173 of Y-Sat), 5'-ATGCAGAGCTG AAAAAGTCACTTB-3';

Y-Sat antisense probe (nucleotides 173-193 of Y-Sat), 5'-GTGAC TTTTTCAGCTCTGCATTTB-3'; and

Random control sequence, 5'-ATGAGCGGAGATAGGCTGGTT CTB-3'.

\section{Analysis of small RNA sequence}

Applied Biosystems sequencing chromatograms were manually reviewed by using CHROMAS (www.technelysium.com.au/chromas.html). All files having unambiguous sequences within the cloning region were analyzed by Ebbie (Ebhardt et al. 2006).

\section{SUPPLEMENTAL MATERIAL}

Supplemental material can be found at http://www.rnajournal.org.

\section{ACKNOWLEDGMENTS}

H.A.E. is currently supported by a post-doctoral fellowship from the Alberta Cancer Research Institute and the Alberta Cancer Foundation. We thank Ming-Bo Wang (Commonwealth Scientific and Industrial Research Organization, Canberra, Australia) for the
HC-Pro RNA samples and sequencing of the Y-Sat genome. This work was supported by grants from the National Science and Engineering Research Council of Canada, the Michael Smith Foundation for Health Research and a postgraduate scholarship from the Natural Sciences and Engineering Council of Canada (to H.A.E.).

Received June 24, 2008; accepted January 9, 2009.

\section{REFERENCES}

Alwine, J.C., Kemp, D.J., and Stark, G.R. 1977. Method for detection of specific RNAs in agarose gels by transfer to diazobenzyloxymethyl-paper and hybridization with DNA probes. Proc. Natl. Acad. Sci. 74: 5350-5354.

Alwine, J.C., Kemp, D.J., Parker, B.A., Reiser, J., Renart, J., Stark, G.R., and Wahl, G.M. 1979. Detection of specific RNAs or specific fragments of DNA by fractionation in gels and transfer to diazobenzyloxymethyl paper. Methods Enzymol. 68: 220-242.

Dressler, D. 1970. The rolling circle for $\phi X$ DNA replication. II. Synthesis of single-stranded circles. Proc. Natl. Acad. Sci. 67: 19341942.

Ebhardt, H.A., Thi, E.P., Wang, M.B., and Unrau, P.J. 2005. Extensive $3^{\prime}$ modification of plant small RNAs is modulated by helper component-proteinase expression. Proc. Natl. Acad. Sci. 102: 13398-13403.

Ebhardt, H.A., Wiese, K.C., and Unrau, P.J. 2006. Ebbie: Automated analysis and storage of small RNA cloning data using a dynamic web server. BMC Bioinformatics 7: 185. doi: 10.1186-1471-2105-7-185.

Eklund, A.C., Turner, L.R., Chen, P., Jensen, R.V., deFeo, G., Kopf-Sill, A.R., and Szallasi, Z. 2006. Replacing cRNA targets with cDNA reduces microarray cross-hybridization. Nat. Biotechnol. 24: 1071-1073.

Horwich, M.D., Li, C., Matranga, C., Vagin, V., Farley, G., Wang, P., and Zamore, P.D. 2007. The Drosophila RNA methyltransferase, DmHen1, modifies germline piRNAs and single-stranded siRNAs in RISC. Curr. Biol. 17: 1265-1272.

Jung, J.H. and Park, C.M. 2007. MIR166/165 genes exhibit dynamic expression patterns in regulating shoot apical meristem and floral development in Arabidopsis. Planta 225: 1327-1338.

Kim, I., McKenna, S.A., Viani Puglisi, E., and Puglisi, J.D. 2007. Rapid purification of RNAs using fast performance liquid chromatography (FPLC). RNA 13: 289-294.

Li, J., Yang, Z., Yu, B., Liu, J., and Chen, X. 2005. Methylation protects miRNAs and siRNAs from a $3^{\prime}$-end uridylation activity in Arabidopsis. Curr. Biol. 15: 1501-1507.

MAQC ConsortiumShi, L., Reid, L.H, Jones, W.D, Shippy, R., Warrington, J.A., Baker, S.C., Collins, P.J., de Longueville, F., Kawasaki, E.S. et al. 2006. The Microarray quality control (MAQC) project shows inter- and intraplatform reproducibility of gene expression measurements. Nat. Biotechnol. 24: 1151-1161.

Masuta, C., Suzuki, M., Kuwata, S., Takanami, Y., and Koiwai, A. 1993. Yellow mosaic symptoms induced by Y-satellite RNA of cucumber mosaic virus is regulated by a single incompletely dominant gene in wild Nicotiana species. Phytopathology 83: 411-413.

Mi, S., Cai, T., Hu, Y., Chen, Y., Hodges, E., Ni, F., Wu, L., Li, S., Zhou, H., Long, C., et al. 2008. Sorting of small RNAs into arabidopsis argonaute complexes is directed by the $5^{\prime}$ terminal nucleotide. Cell 133: 116-127.

Modahl, L.E. and Lai, M.M. 1998. Transcription of hepatitis delta antigen mRNA continues throughout hepatitis delta virus (HDV) replication: A new model of HDV RNA transcription and replication. J. Virol. 72: 5449-5456.

Morin, R.D., Aksay, G., Dolgosheina, E., Ebhardt, H.A., Magrini, V., Mardis, E.R., Sahinalp, S.C., and Unrau, P.J. 2008. Comparative analysis of the small RNA transcriptomes of pinus contorta and oryza sativa. Genome Res. 18: 571-584. 
Rajagopalan, R., Vaucheret, H., Trejo, J., and Bartel, D.P. 2006. A diverse and evolutionarily fluid set of microRNAs in arabidopsis thaliana. Genes \& Dev. 20: 3407-3425.

Sharbati-Tehrani, S., Kutz-Lohroff, B., Bergbauer, R., Scholven, J., and Einspanier, R. 2008. miR-Q: A novel quantitative RT-PCR approach for the expression profiling of small RNA molecules such as miRNAs in a complex sample. BMC Mol. Biol. 9: 34. doi: 10.1186/1471-2199-9-34.

Sivakumaran, K. and Kao, C.C. 1999. Initiation of genomic plusstrand RNA synthesis from DNA and RNA templates by a viral RNA-dependent RNA polymerase. J. Virol. 73: 6415-6623.

Sleat, D.E., Zhang, L., and Palukaitis, P. 1994. Mapping determinants within cucumber mosaic virus and its satellite RNA for the induction of necrosis in tomato plants. Mol. Plant Microbe Interact. 7: 189-195.
Takanami, Y. 1981. A striking change in symptoms on cucumber mosaic-virus-infected tobacco plants induced by a satellite RNA. Virology 109: 120-126.

Wang, M.B., Bian, X.Y., Wu, L.M., Liu, L.X., Smith, N.A., Isenegger, D., Wu, R.M., Masuta, C., Vance, V.B., Watson, J.M., et al. 2004. On the role of RNA silencing in the pathogenicity and evolution of viroids and viral satellites. Proc. Natl. Acad. Sci. 101: 3275-3280.

Yang, Z., Ebright, Y.W., Yu, B., and Chen, X. 2006. HEN1 recognizes 21-24 nt small RNA duplexes and deposits a methyl group onto the $2^{\prime} \mathrm{OH}$ of the $3^{\prime}$ terminal nucleotide. Nucleic Acids Res. 34: 667-675.

Zhang, J., Finney, R.P., Clifford, R.J., Derr, L.K., and Buetow, K.H. 2005. Detecting false expression signals in high-density oligonucleotide arrays by an in silico approach. Genomics 85: 297-308. 

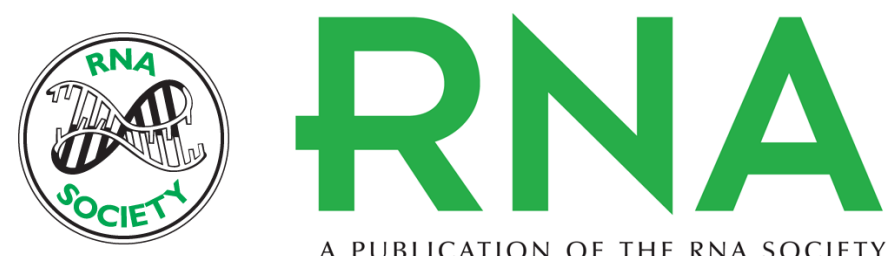

A PUBLICATION OF THE RNA SOCIETY

\section{Characterizing multiple exogenous and endogenous small RNA populations in parallel with subfemtomolar sensitivity using a streptavidin gel-shift assay}

H. Alexander Ebhardt and Peter J. Unrau

RNA 2009 15: 724-731 originally published online February 23, 2009

Access the most recent version at doi:10.1261/rna.1235109

Supplemental Material

References

License

Email Alerting Service
http://rnajournal.cshlp.org/content/suppl/2009/02/23/rna.1235109.DC1

This article cites 23 articles, 9 of which can be accessed free at: http://rnajournal.cshlp.org/content/15/4/724.full.html\#ref-list-1

Receive free email alerts when new articles cite this article - sign up in the box at the top right corner of the article or click here. 